\title{
Il ruolo dei nuovi porti e delle nuove fortificazioni cinquecentesche nella trasformazione dell'immagine delle città mediterranee
}

The role of the new sixteenth century harbours and fortifications on the transformation of the Mediterranean city's image

\section{Ludovico Micara}

Università degli Studi dell'Adriatico “G. d'Annunzio” Chieti-Pescara, Pescara, Italy, ludovico.micara@gmail.com

\begin{abstract}
The richness of historical heritage and the complex stratification of sites, combined with the defensive functions and techniques of the fortifications, confer an added value to the cities overlooking the Mediterranean Sea. The fortifications and the new harbours of Tripoli, Libya and Alexandria, Egypt are not isolated structures, which just enhance the qualities and visibility of the places where they are built. Rather, as they come into contact with the pre-existing ancient urban systems, harbours and fortifications contribute to enriching the architectural qualities and values of the cities. In such situations, the new defensive interventions assume a strong transformational role in relation with the sites where they are located, and thus create new urban realities, and new meanings to be deciphered. In the discovery of these new interpretive possibilities, lies the fascination these studies can still offer today.
\end{abstract}

Keywords: Sixteenth century harbours and fortifications, Mediterranean city, Tripoli, Alexandria.

\section{Introduzione}

I sistemi difensivi e le fortificazioni sono state da sempre un potente elemento di disegno e di caratterizzazione del paesaggio. La messa in campo di strumenti atti a individuare e ad utilizzare le geometrie palesi o nascoste dei siti, sia che riguardasse la difesa di insediamenti urbani o di emergenti siti isolati, ha permesso il loro ridisegno in funzione strategica, insieme alla formalizzazione di una nuova più astratta immagine rispetto a quella naturale, e di un nuovo paesaggio artificiale.

Questo è tanto più vero dal momento che, con lo sviluppo, dal XVI secolo in poi, dei nuovi trattati scientifici sulla balistica delle armi da fuoco, da parte degli ingegneri militari, si diffondono i sistemi di difesa bastionati, molto più impattanti rispetto ai precedenti sistemi difensivi. La loro

messa in opera sulle coste del Mediterraneo, nell'ambito della Reconquista, da parte di Carlo $\mathrm{V}$, delle città già occupate dagli arabi, produce una generale trasformazione del paesaggio costiero, che mette in secondo piano i profili delle medine e dei centri antichi, che un tempo lo avevano caratterizzato.

Il caso di Tripoli in Libia, come di altre città storiche, come Alessandria d'Egitto, la cui formazione risale a civiltà urbane anteriori o contemporanee all'Islam, illustra un ulteriore capitolo di questa affascinante storia. Le nuove strutture difensive si innestano così su strutture urbane complesse, ricche di tracce archeologiche forti, evidenti anche nelle rigenerazioni arabo-islamiche, ma estranee alle "moderne" geometrie dei nuovi sistemi bastionati, con i quali vengono a patti, 


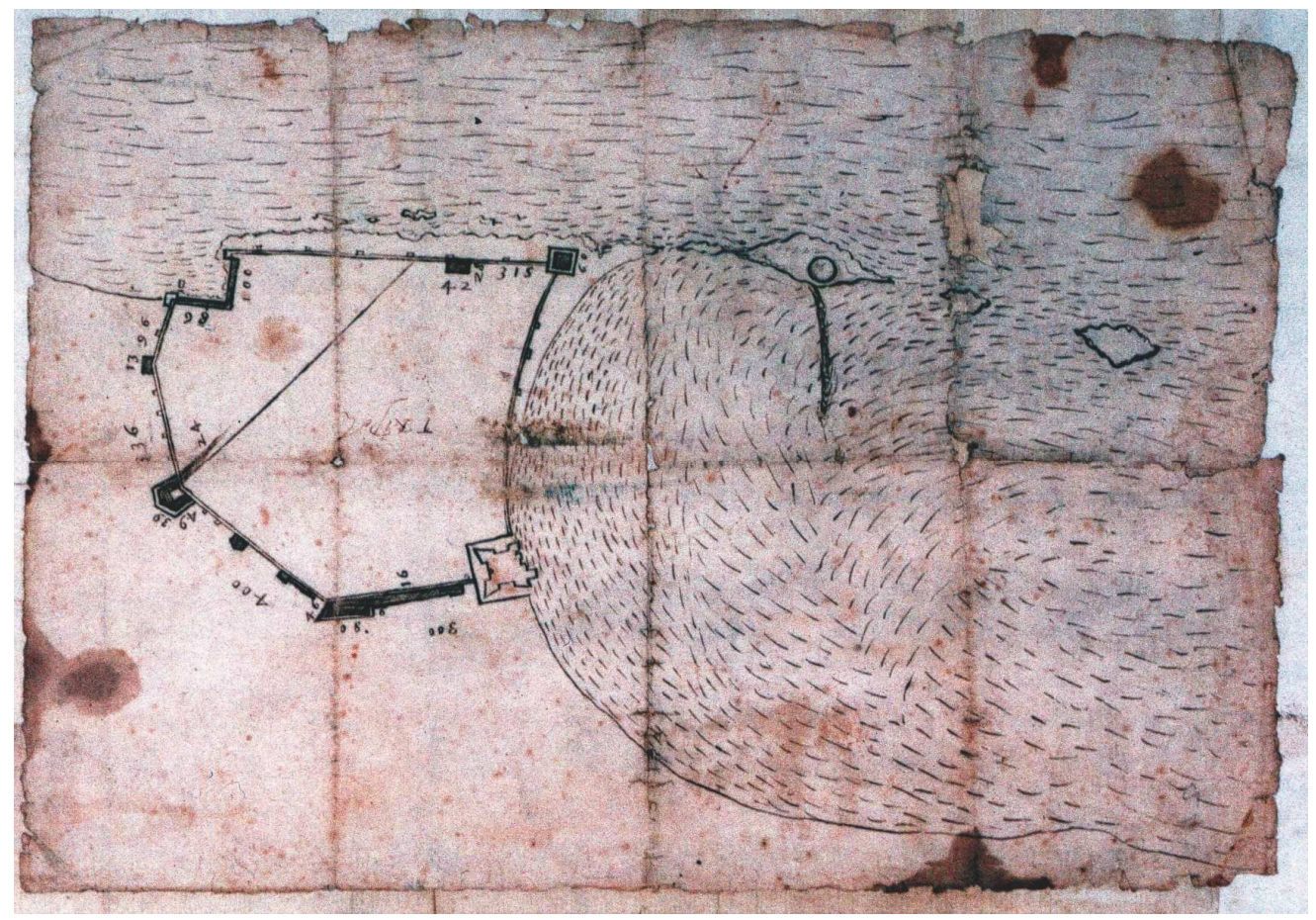

Fig. 1. Le fortificazioni di Tripoli (Archivio di Stato di Torino, Architettura Militare, vol. II, f. 39v).

creando inediti compromessi e configurazioni innovative.

\section{Tripoli}

Un disegno nelle Biblioteca Antica dell'Archivio di Stato di Torino rappresenta il sistema difensivo della città di Tripoli nel 1559, ma non la Medina in esso contenuta. Il disegno rappresenta la situazione delle mura e del porto di Tripoli subito dopo il dominio spagnolo (1510-1530) e dei Cavalieri di Malta (1530-1551).

Riconquistata la città nel 1551 dalla flotta ottomana comandata da Sinan Pasha, rinforzata dai combattenti arabi di Murad Agha, il nuovo governatore di Tripoli Darghut Pasha, che aveva favorito, con le sue navi corsare, la riconquista, nel timore di un nuovo attacco da parte dell' armata cristiana, si dedicò al rafforzamento delle difese militari e delle mura. Risalgono a questo decennio alcune nuove strutture difensive che compaiono nel disegno e ne suggeriscono una probabile datazione.
Tra esse il Castello bastionato (Saray al-Hamra il Forte Rosso), nell' angolo meridionale delle mura pentagonali, circondato da un fossato, e sul lato opposto, all'estremità nord delle mura, un forte a pianta quadrata, detto di San Pietro, voluto da Darghut Pasha nel 1559, quando temeva di essere attaccato dall' armata di Spagna. Un ulteriore fortilizio, in alto a destra nella tavola, chiamato Castellejo e più tardi Burg el-Mandrik viene costruito sugli scogli che proteggono a nord-est il porto stesso, con un nuovo molo per l'attracco delle navi.

Nell'immagine delle fortificazioni di Tripoli non è rappresentata la Medina. Dobbiamo ricorrere ad immagini più recenti per capire il rapporto tra tali fortificazioni, l'abitato al loro interno e l'impatto con i nuovi recenti contesti urbani creati dalle espansioni coloniali e moderne all'esterno della Medina. Un nuovo fronte-mare, il lungomare Volpi, opera di Armando Brasini, ingloba l'immagine della città mediterranea. 
Ma un'altra conseguenza delle trasformazioni prodotte dal contatto tra le fortificazioni delle medine giunte fino a noi, e i contesti urbani contemporanei sono gli spazi di risulta tra questi diversi sistemi.

Tali spazi, un tempo considerati "vuoti", senza una precisa funzione e destinati agli usi più diversi, sono oggi considerati "spazi pubblici" di grande importanza per gli assetti urbani contemporanei e per determinare l'immagine della città mediterranea. Tra essi il vuoto tra il castello e la città coloniale, la cosiddetta Piazza Verde, teatro delle grandi adunate volute da Gheddafi, e la nuova vista delle mura non più come un tempo arrivando in nave dal mare, ma dalla nuova strada di circonvallazione della Medina.

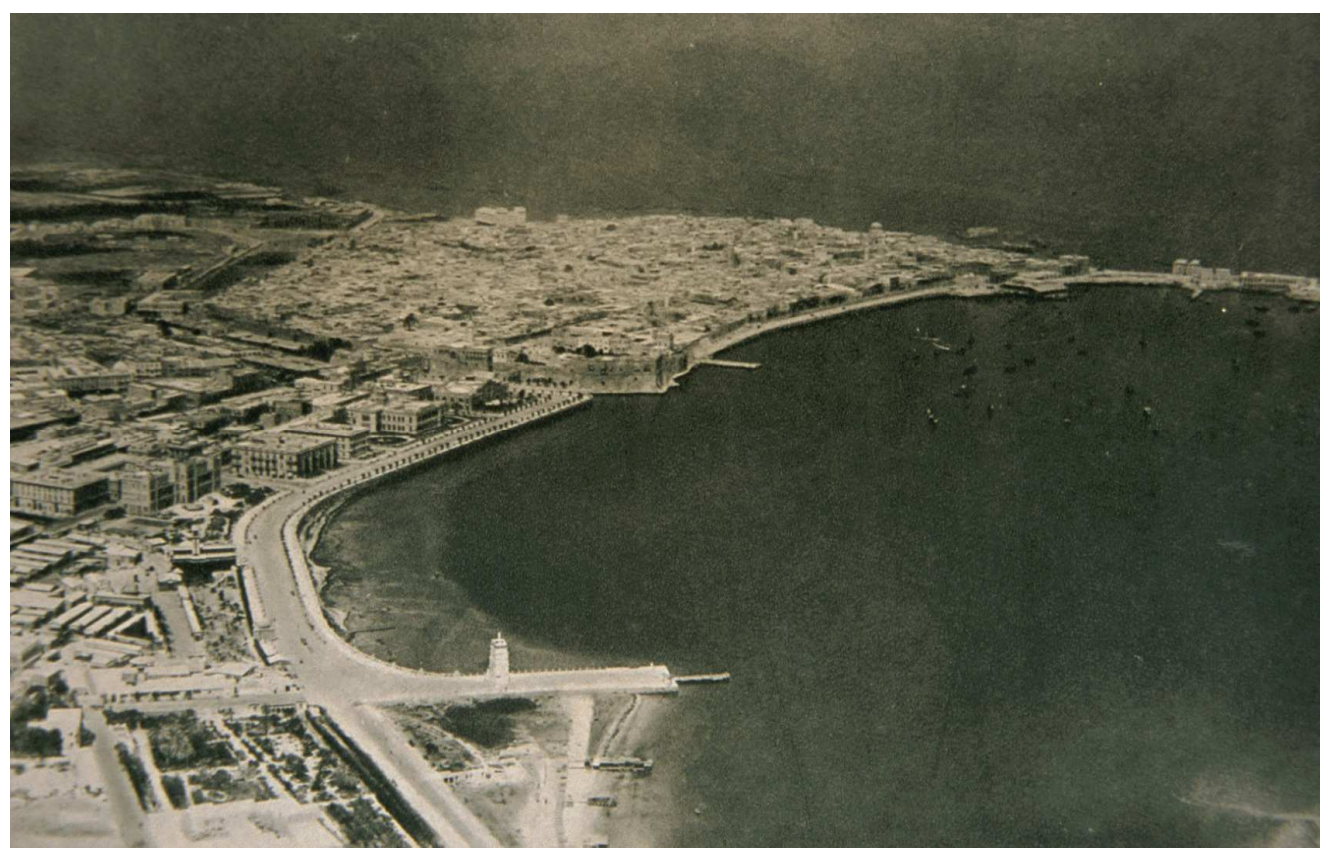

Fig. 2. La Medina di Tripoli, (Al Bayda, la Città Bianca) e il lungomare Volpi (1930).

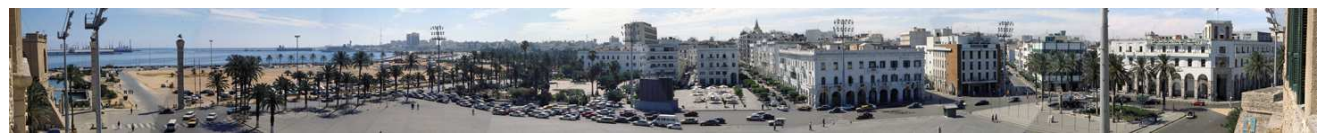

Fig. 3. La cosiddetta Piazza Verde vista dal Castello verso la città coloniale.

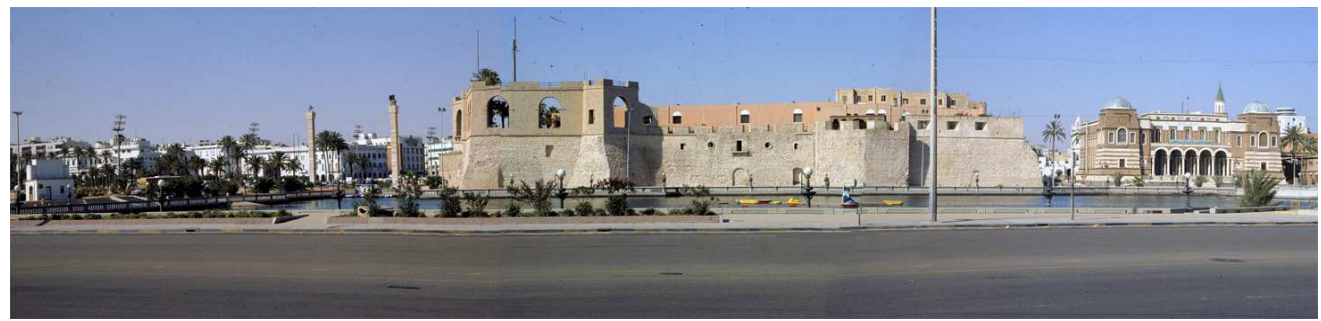

Fig. 4. Veduta del Castello e della piazza Verde dalla nuova strada di circonvallazione della Medina. 


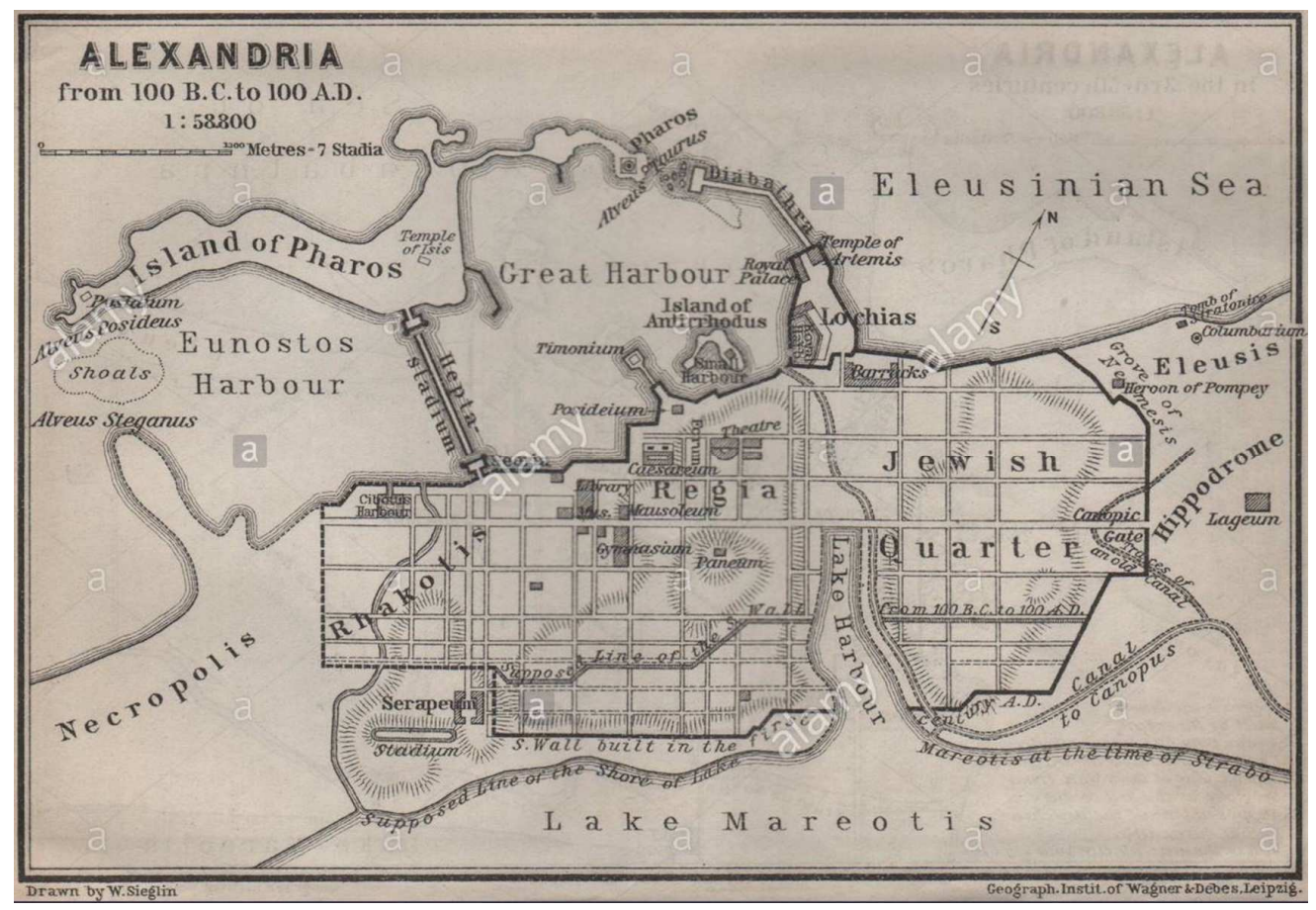

Fig. 5. Alessandria: la città antica.

\section{Alessandria}

La struttura ellenistica della città antica fondata da Alessandro Magno nel 332 a.C., progettata da Dinocrate di Rodi secondo un impianto ippodameo basato sul grande asse della via Canopica, orientato est-ovest, è circondata da mura un tempo rafforzate da numerose torri. La città si apriva all'esterno attraverso quattro porte.

La Porta Del Borgo a nord (la Bab al-Bahr o Porta del Mare della città conquistata nel 642 dagli Arabi, Al-Iskandariyya) portava all'Eptastadion, il ponte-diga lungo sette stadi che collegava la città ellenistica all'isola del Faro e divideva i due grandi porti naturali: orientale, Porto grande (Portus Magnus), e occidentale, Porto vecio (Eunostus).

La Porta Dello Pepe a sud (Bab Sidra, o Porta delle Spezie nelle fonti occidentali) costituiva l'ingresso attraverso cui entravano in città le carovane provenienti dal Maghreb e dall'Egitto interno.

La Porta Di Rossetto ad est (Bab Rashid o Porta di Rosetta) conduceva a Rosetta; mentre la Porta della Dogana, ancora a nord (probabilmente la Bab al-Akhdar o Bab al-Khidr), prospiciente il Porto grande, oltre ad essere riservata all'uso della Dogana, conduceva ad uno dei tre grandi cimiteri della città ed era aperta solo una volta alla settimana, il Venerdì, per i visitatori.

Al di là della Porta Del Borgo, verso nord, l'antico Eptastadion, insabbiato e trasformatosi in una penisola, terminante nel sito dell'ormai caduto Pharos, venne nel tempo densamente costruito.

Sarà proprio il Borgo la parte di Alessandria che, secondo la rappresentazione della città nella $D e$ scription de l'Egypte napoleonica (1800) riuscirà a sopravvivere alla decadenza ed alla rovina della città murata, prima della rinascita ottocentesca dovuta a Mohamed Alì.

Come possiamo vedere da questa immagine e dalla tuttora simile odierna veduta satellitare, è ancora a partire dai nuovi porti e dalle fortificazioni urbane che nasce e si trasforma la nuova città.

L'entrata del Porto grande è protetta nel suo lato occidentale dal Faraglione Grande, la fortezza- 
faro costruita dal Sultano Ka'it Bay nel 1447 sulle rovine dell'antico Pharos di Alessandria Quest'ultimo edificio, una delle meraviglie del mondo antico, sopravvisse alla conquista araba per diversi secoli, chiamato manara, manar (minareto) o fanar (fanale, faro), finché, distrutto nel corso di vari terremoti, non venne ricostruito come forte a guardia del cosiddetto Porto novo.

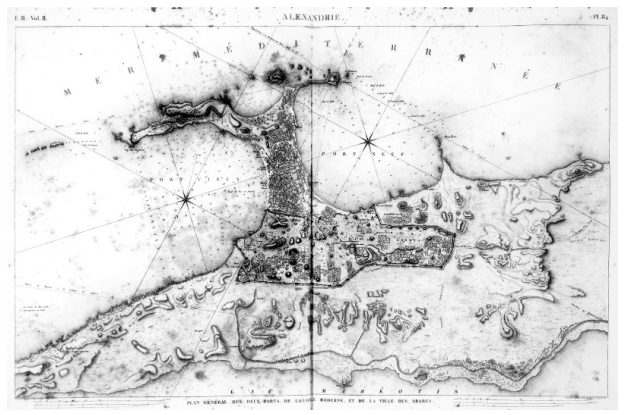

Fig. 6. Alessandria (Description de l'Egypte, vol. II, planche 84, Paris 1809).

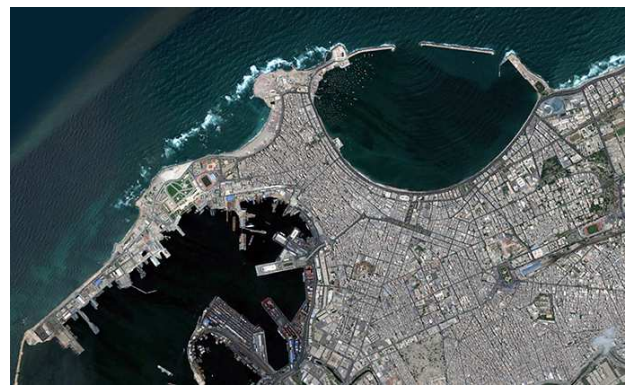

Fig. 7. Veduta satellitare di Alessandria d'Egitto.

La fortezza si compone di una Citadela, piattaforma fortificata con torri e bastioni, su cui si erge il Maschio a pianta quadrata con quattro torri circolari agli angoli.

Il disegno che ne fa il viaggiatore ligure Pellegrino Brocardi in occasione del suo viaggio in Egitto, tappa di un pellegrinaggio in Terra Santa nel 1556, è molto simile a quello che verrà rilevato durante la Campagna d'Egitto napoleonica e pubblicato nella Description de l'Egypte, dove la variazione più importante sembra essere la porta fortificata, nell'angolo sud-est, che protegge l'ingresso alla cittadella dal ponte di collegamento con la terra ferma e con il Borgo.

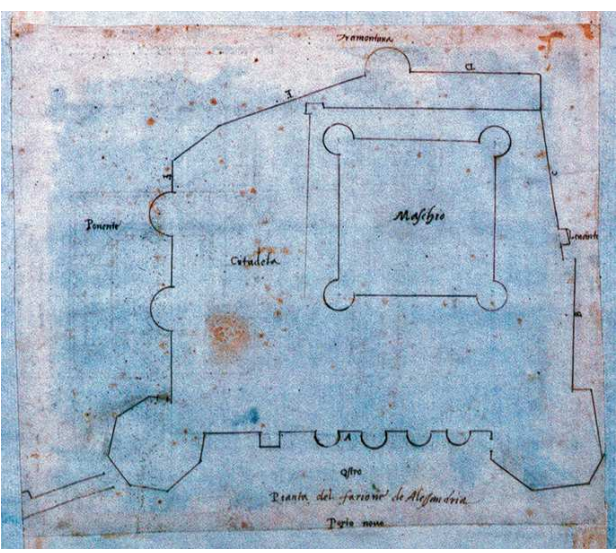

Fig. 8. Pellegrino Brocardi, Pianta del Farione de Alessandria (Archivio di Stato di Torino, Architettura Militare, vol. II, f. $43 v$ ).

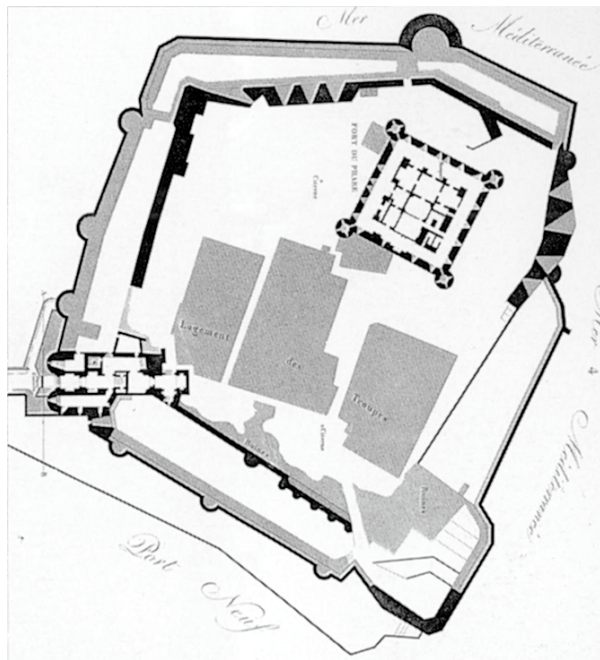

Fig. 9. Alessandria, pianta della Fortezza-faro di Ka'it Bay (Description de l'Egypte, vol. II, planche 87, Paris 1809).

$\mathrm{Si}$ viene dunque a configurare, attraverso questa fortezza, sostituto monumentale dell' antico e mitico Faro, e assieme a quanto rimane della città antica sull'altra sponda, un nuovo paesaggio urbano in cui il Porto grande o Porto nuovo assume, aldilà della funzione nautica che gli compete, il ruolo di nuovo grande spazio pubblico sul mare.

L'aspetto di questo spazio non doveva essere molto diverso dalla bella veduta riportata dalla Description napoleonica. 


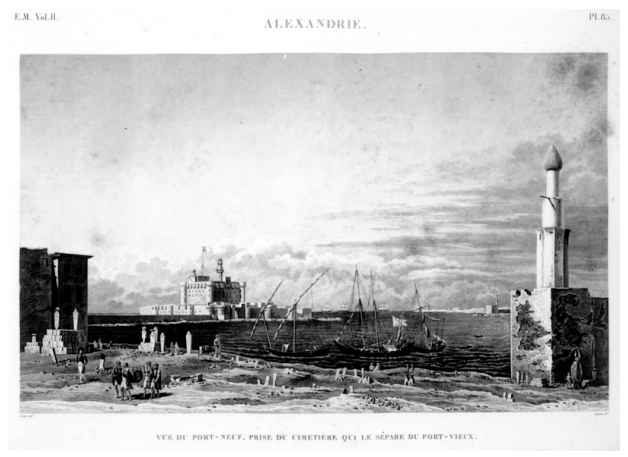

Fig. 10. Alessandria, veduta del Porto Nuovo (Description de l'Egypte, vol. II, planche 85, Paris 1809).

I resti e le rovine del cimitero arabo, in primo piano, come gli edifici e i monumenti della civiltà egizia testimoniano della compresenza di storie diverse, della sovrapposizione di tracce urbane e monumentali che, accanto ai nuovi interventi difensivi, esprimono la qualità insediativa dei centri urbani fortificati nelle coste del Mediterraneo.

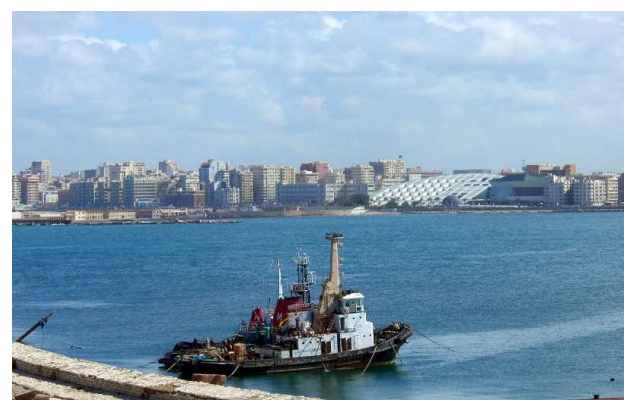

Fig. 11. Veduta della nuova Biblioteca di Alessandria dalla Fortezza d Ka'it Bay.

Non è quindi un caso che nel 1988, nel momento in cui il governo egiziano decide di ricostruire la nuova Biblioteca di Alessandria, erede dell'antica, prestigiosa Bibliotheca Alexandrina, distrutta da un incendio, sembra provocato da Giulio Cesare nel 48 a.C., si sia scelto di costruirla in un bellissimo sito nel centro della città, all'estremità orientale del Porto Grande o Porto nuovo, con vista sulla Fortezza-faro di Qa'yt Bay. A conferma ancora della continuità insediativa e della permanenza di valori urbani nelle città storiche del Mediterraneo.

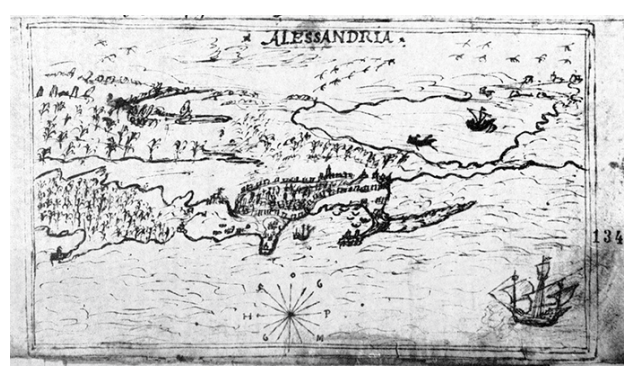

Fig. 12. Pellegrino Brocardi, Alessandria schizzo (Biblioteca Apostolica Vaticana, Codice Vaticano Latino 6038, ff. 130-140).

\section{Conclusione}

Nuovi porti e nuove fortificazioni sono certamente un potente elemento di qualificazione dei paesaggi costieri del Mediterraneo. Non solo come strutture isolate, che esaltano le qualità e le visibilità dei luoghi dove sono edificate, ma anche come strutture che entrano in relazione con sistemi già esistenti, di cui arricchiscono le qualità e potenzialità architettoniche. Spesso sorgono accanto o sopra insiemi urbani, a volte antichi e ricchi di storia, come nei casi descritti in precedenza.

In tali situazioni i nuovi interventi difensivi trasformano i luoghi e creano inedite realtà, nuove trame, con significati spesso da decifrare.

Nella scoperta di tali possibilità, nella interpretazione di tali significati risiede il fascino che questi studi ancora riservano.

\section{Bibliography}

Aurigemma, S. (1916). "Le fortificazioni della città di Tripoli”, Notiziario Archeologico del Ministero delle Colonie, II, I-II, pp. 217-300.

Aurigemma, S. (1929). "Le fortificazioni di Tripoli in antiche vedute del seicento e del settecento", Rivista delle Colonie Italiane, III, 11, pp. 1104-1128; III, 12, pp. 1217-1237.

Fraser, P.M. (1986). Ptolemaic Alexandria, Ed. Oxford, I, ill. 3, p. 20-26. 
Labib, S. (s.d.). “Al-Iskandariyya”, in Islamic Encyclopaedia, New Edition, p. 133.

Mahmoud Bey, el-F. (1872). Mémoire sur l'antique Alexandrie, Ed. Copenhague.

Micara, L. (2008a). “Alessandria, f. 40”, in Architettura Militare II, Ministero per i Beni e le Attività Culturali Direzione Generale per gli Archivi Ed., Roma., pp. 46, 115-116.

Micara, L. (2008b). "Pianta del farione de Alessandria, f. 43v", in Architettura Militare II, Ministero per i Beni e le Attività Culturali - Direzione Generale per gli Archivi Ed., Roma., pp. 51, 118.

Micara, L. (2008c). "The Ottoman Tripoli: a Mediterranean Medina”, in Jayyushi, S.K.; Holod, R.; Petruccioli, A.; Raymond, A., eds., The City in the Islamic World, Brill Ed., Leiden-Boston, pp. 383-406.

Micara, L. (2013). Tripoli a Mediterranean Medina, Gangemi Ed., Roma.

Micara, L.; Vadini, E. (2006). "Tripoli and its Seafront”, in Città di Pietra/ Cities of Stone, Biennale di Venezia Marsilio Ed., Venezia, pp. 184-189.

Rossi, E. (1968). Storia di Tripoli e della Tripolitania dalla conquista araba al 1911, Istituto per l'Oriente Ed., Roma.

Talamona, M. (1993). “Città europea e città araba in Tripolitania”, in Gresleri, G., ed., L'architettura italiana d'oltremare, Marsilio Ed., Venezia, pp. 257-277.

Von Henneberg, K. (1994). "Piazza Castello and the Making of a Fascist Capital”, in Çelik, Z.; Favro, D.; Ingersoll, R., eds., Streets, Critical Perspectives on Public Space, University of California Press Ed. 
